\title{
Predictors of obstructive sleep apnea in males with metabolic syndrome
}

\author{
This article was published in the following Dove Press journal: \\ Vascular Health and Risk Management \\ 16 April 2010 \\ Number of times this article has been viewed
}

\author{
Nikolaos Papanas' \\ Paschalis Steiropoulos ${ }^{2}$ \\ Evangelia $\mathrm{Nena}^{2}$ \\ Argyris Tzouvelekis ${ }^{2}$ \\ Athanasios Skarlatos ${ }^{2}$ \\ Maria Konsta ${ }^{2}$ \\ Vasileios Vasdekis ${ }^{3}$ \\ Efstratios Maltezos ${ }^{1}$ \\ Demosthenes Bouros ${ }^{2}$ \\ 'Outpatient Clinic of Obesity, \\ Diabetes and Metabolism, Second \\ Department of Internal Medicine, \\ Medical School, Democritus \\ University of Thrace, Alexandroupolis, \\ Greece; ${ }^{2}$ Sleep Laboratory, \\ Department of Pneumonology, \\ Medical School, Democritus \\ University of Thrace, Alexandroupolis, \\ Greece; ${ }^{3}$ Department of Statistics, \\ Athens University of Economic and \\ Business, Athens, Greece
}

\begin{abstract}
The aim of the present study was to examine the prevalence of metabolic syndrome (MS) and its components among obstructive sleep apnea (OSA) patients vs controls, as well as to investigate which of these components are strongly associated with the presence of OSA in subjects reporting symptoms indicating sleep-disordered breathing. Included were 83 consecutive male subjects, without known concomitant diseases, who visited an outpatient clinic of obesity, diabetes and metabolism. Based on polysomnography, these were divided into two groups: OSA patients $(n=53)$ and controls $(n=30)$. Parameters indicating MS, according to the NCEP ATP III criteria (blood pressure, waist circumference, glucose, triglycerides, and HDL-cholesterol levels) were evaluated in both groups. The criteria for MS were fulfilled in 49 participants. Presence of MS was significantly correlated with the presence of OSA. However, after adjustment for BMI, only serum glucose was significantly associated with the presence of OSA $(P=0.002)$. Conversely, the presence of MS was associated with a significant reduction in percentage of slow-wave sleep $(P=0.030)$. In conclusion, these results provide further evidence for the association between OSA and MS. Between subjects with MS, elevated serum glucose levels indicate a higher probability for the presence of OSA.
\end{abstract}

Keywords: diabetes mellitus, glucose, metabolic syndrome, obstructive sleep apnea

\section{Introduction}

Obstructive sleep apnea (OSA) is a common disorder characterized by repetitive episodes of partial or complete obstruction of the upper airway during sleep, ultimately leading to increased respiratory effort, oxyhemoglobin desaturation, sleep fragmentation, and excessive daytime sleepiness. ${ }^{1,2}$ Several studies have investigated the association of OSA with cardiovascular morbidity and mortality, systemic inflammation and impaired glucose metabolism..$^{2-12}$

Metabolic syndrome (MS) is a combination of factors, including obesity with central adiposity, glucose intolerance, dyslipidemia, and hypertension, predisposing patients to the development of cardiovascular disease and diabetes. ${ }^{13-15}$ Its prevalence tends to increase worldwide due to the increase of obesity. ${ }^{13,16}$ The criteria for the diagnosis of metabolic syndrome vary according to the definition but, in all definitions, the key components are obesity, impaired glucose metabolism, dyslipidemia and hypertension. ${ }^{14,17}$

The correlation between OSA and MS is complex and incompletely understood. ${ }^{18,19}$ Indeed, the independent association between OSA, insulin resistance, and the development of type 2 diabetes has been established in numerous works. ${ }^{3,5,6,9,20-22}$ Thus, OSA has been proposed as an extra component of MS, with intermittent hypoxia being identified as a potential triggering factor. ${ }^{3,20}$ However, the evidence for the contribution
Correspondence: Paschalis Steiropoulos Medical School, Democritus University of Thrace, Alexandroupolis, 68100, Greece

$\mathrm{Tel} / \mathrm{Fax}+30255 \mathrm{I} 075096$

Email steiropoulos@yahoo.com 
of OSA to the development of MS is far from conclusive. Previous studies in European and East-Asiatic populations have demonstrated an independent association between OSA and the MS. ${ }^{20,23-25}$ These studies have used either the National Cholesterol Educational Program - Adult Treatment Program (NCEP-ATP) III criteria or the definition of the International Diabetes Federation (IDF). ${ }^{14,17}$

Therefore, the aim of the present study was to examine the prevalence of MS and its components among OSA patients vs. controls, as well as to investigate which of these components are strongly associated with the presence of OSA in subjects reporting symptoms indicative of sleep-disordered breathing.

\section{Materials and methods Study group}

The present study included 83 male subjects, without previous diagnosis or treatment for OSA, who were referred to the Sleep Laboratory of the Department of Pneumonology of the Democritus University of Thrace, Greece, between January and June 2006, reporting symptoms indicating sleep-disordered breathing (eg, excessive daytime sleepiness, snoring, and/or morning headaches, fatigue). These were consecutively recruited from the Outpatient Clinic of Obesity, Diabetes and Metabolism in the Second Department of Internal Medicine of the same institution. Exclusion criteria were as follows: previously-diagnosed diabetes mellitus (DM); arterial hypertension; any cardiovascular or cerebrovascular disease; liver, endocrine, inflammatory, or other chronic disease; systemic medication use; smoking. The study was conducted in accordance with the Helsinki Declaration of Human Rights and all subjects gave their informed consent.

\section{Study design}

\section{Initial assessment}

At first visit, medical history was recorded and physical examination was performed. Anthropometrical data (age, sex, neck, waist and hip circumferences) along with daytime habits (such as smoking, alcohol use, exercise) were recorded. Weight and height were measured to the nearest kilogram and centimeter respectively, and body mass index (BMI) was calculated $\left(\mathrm{BMI}=\right.$ weight $/$ height $\left.^{2}\right)$. During examination, participants were lightly clothed, without shoes. Neck circumference was measured at the cricothyroid level, waist circumference in the middle between the 12 th rib and the iliac crest, and hip circumference at the level of great trochander, by measuring tape. Diabetes was defined by fasting hyperglycemia above $126 \mathrm{mg} / \mathrm{dL} \cdot{ }^{26}$ Diagnosis of arterial hypertension was based either on antihypertensive treatment with medical history of documented high blood pressure, or on measurement of systolic pressure $\geq 140 \mathrm{mmHg}$ and/or measurement of diastolic pressure $\geq 90 \mathrm{mmHg}$ on three separate occasions. ${ }^{27}$ Diagnosis of MS was based on the NCEP-ATP III criteria. ${ }^{17}$ Sleepiness was evaluated by the Greek version of the Epworth Sleepiness Scale (ESS). ${ }^{28}$

\section{Polysomnography (PSG)}

All subjects underwent an attended overnight polysomnography (Alice ${ }^{\circledR}$ 4, Philips Respironics, Murrysville, PA, USA) using a standard montage of electroencephalogram (EEG), electroocculogram, electromyogram (EMG), and electrocardiogram (ECG) signals, together with pulse oxymetry and airflow, detected using combined oronasal thermistors. Thoracic cage and abdominal motion were recorded by inductive plethysmography. EEG recordings were manually scored according to standard criteria. Apnea was defined as complete cessation of airflow for $\geq 10 \mathrm{sec}$; hypopnea as a $50 \%$ reduction in airflow for $\geq 10 \mathrm{sec}$, accompanied by $\geq 4 \%$ desaturation or by an EEG-recorded arousal. Apnea Hyponea Index (AHI) was defined as the number of apneas and hypopneas per hour of polysomnographically-recorded sleep time, while Oxygen Desaturation Index (ODI) was defined as the number of oxyhemoglobin desaturations $\geq 3 \%$ per hour of polysomnographically recorded sleep time.OSA was defined as AHI $>5$, plus daytime symptoms. PSG was performed from 2200 to 0600 hours. Patients with pure or mainly central apneas were excluded from the study.

\section{Biochemical analysis of blood samples}

Blood samples were obtained the morning after polysomnography, between 0800 and 0900 hours, following an overnight fast. Total, high-density lipoprotein (HDL)- and low-density lipoprotein (LDL)-cholesterol and triglyceride levels were measured by enzymatic colorimetric methods, while glucose was measured by enzymatic method (Olympus AU640 ${ }^{\mathrm{TM}}$, Olympus Diagnostica GmbH, Hamburg, Germany).

\section{Statistical analysis}

Descriptive statistics are expressed as mean \pm standard deviation (SD). Chi-squared $\left(\chi^{2}\right)$ tests were used for exploring the relationship between two categorical variables. The association between the presence of metabolic syndrome and the presence of apnea, adjusted for BMI, was determined using logistic regression. The same technique was used for determining the relationship between the presence of metabolic 
syndrome and other explanatory variables, either factors or covariates. The results are presented as regression coefficients or Wald tests, along with their statistical significance. All analyses were performed using SPSS v.13 (Statistical Package for Social Sciences, Chicago, IL, USA).

\section{Results}

Based on polysomnography, subjects were divided into OSA patients ( $n=53$; mean AHI 57.2 \pm 26.8 events/hour) and healthy controls ( $n=30$; mean AHI $3.5 \pm 1.9$ events/hour). General and sleep characteristics of both groups are presented in Table 1.

Among the 83 participants, 49 (59\%) filled the criteria for MS. Specifically, MS was present in $71.7 \%$ of the OSA patients (38 out of 53) and in $36.7 \%$ of the controls (11 out of 30). Despite the strong association between the presence of metabolic syndrome and sleep apnea $\left(\chi^{2}=8.91\right.$, $P=0.003)$, logistic regression analysis showed that this association became marginally non-significant $(\mathrm{B}=-1.081$, $P=0.052$ ) when BMI was taken into account.

Looking separately at each component of the metabolic syndrome, the presence of OSA was associated with serum glucose levels above the cut-off set by NCEP ATP III $\left(\chi^{2}=12.533, P=0.000\right),{ }^{16}$ with waist circumference above the cut-off for each gender $\left(\chi^{2}=9.974, P=0.001\right)$, and blood pressure exceeding the cut-off point $\left(\chi^{2}=3.374, P=0.043\right)$. On logistic regression analysis, however, only the presence of increased serum glucose levels was associated with sleep apnea adjusted for BMI $(B=2.530, P=0.002)$. Table 2 shows the estimated regression coefficients of each parameter of MS for sleep apnea, along with their statistical significance.

Conversely, the investigation of the correlations between sleep or anthropometric characteristics and presence of MS yielded only one significant correlation. There was a significant negative correlation $(\mathrm{B}=-0.079, P=0.030)$

Table I Anthropometric, sleep, and metabolic characteristics of the studied population and comparison between obstructive sleep apnea patients and controls

\begin{tabular}{|c|c|c|c|c|}
\hline Characteristic & Total $(n=83)$ & Controls $(n=30)$ & OSA patients $(n=53)$ & $P$ \\
\hline Age & $45.88 \pm 9.01$ & $45.47 \pm 4.16$ & $46.11 \pm 10.87$ & 0.701 \\
\hline BMI $\left(\mathrm{kg} / \mathrm{m}^{2}\right)$ & $32.29 \pm 6.91$ & $29.04 \pm 4.31$ & $34.13 \pm 7.44$ & 0.001 \\
\hline Neck circumference $(\mathrm{cm})$ & $42.54 \pm 3.22$ & $40.53 \pm 2.4$ & $43.16 \pm 3.19$ & 0.000 \\
\hline Waist circumference $(\mathrm{cm})$ & $113.34 \pm 13.05$ & $105.13 \pm 6.44$ & $114.47 \pm 12.95$ & 0.000 \\
\hline Hip circumference $(\mathrm{cm})$ & $117.47 \pm 19.56$ & $105.93 \pm 7.55$ & $118.55 \pm 19.14$ & 0.000 \\
\hline WHR & $0.97 \pm 0.088$ & $0.99 \pm 0.04$ & $0.98 \pm 0.08$ & 0.254 \\
\hline Systolic blood pressure (mmHg) & $137.26 \pm 14.34$ & $128.37 \pm 7.36$ & $133.28 \pm 8.43$ & 0.009 \\
\hline Diastolic blood pressure $(\mathrm{mmHg})$ & $82.53 \pm 11.44$ & $80.5 \pm 7.22$ & $81.23 \pm 8.85$ & 0.703 \\
\hline ESS & $9.50 \pm 5.95$ & $5.9 \pm 4.52$ & $11.42 \pm 5.78$ & 0.000 \\
\hline $\mathrm{AHI}$ (events/hour) & $37.76 \pm 33.61$ & $3.46 \pm 1.89$ & $57.17 \pm 26.77$ & 0.000 \\
\hline $\mathrm{avSpO}_{2}(\%)$ & $91.41 \pm 4.30$ & $93.68 \pm 1.9$ & $90.12 \pm 4.74$ & 0.000 \\
\hline $\operatorname{minSpO}_{2}(\%)$ & $77.17 \pm 10.82$ & $86.37 \pm 3.24$ & $71.96 \pm 10.12$ & 0.000 \\
\hline TST with $\mathrm{SpO}_{2}<90 \%$ (\%TST) & $22.25 \pm 27.67$ & $4.93 \pm 18.27$ & $58.24 \pm 26.59$ & 0.000 \\
\hline ODI (events/hour) & $38.94 \pm 33.44$ & $4.84 \pm 3.21$ & $58.24 \pm 26.59$ & 0.000 \\
\hline Sleep efficiency (\%TST) & $86.77 \pm 9.92$ & $84.35 \pm 8.87$ & $88.7 \pm 10.39$ & 0.083 \\
\hline Stage I (\%TST) & $10.94 \pm 6.93$ & $8.63 \pm 5.08$ & $12.78 \pm 7.70$ & 0.017 \\
\hline Stage 2 (\%TST) & $49.94 \pm 13.66$ & $44.58 \pm 12.64$ & $54.23 \pm 13.10$ & 0.005 \\
\hline Stage $3+4$ (\%TST) & $15.55 \pm 11.89$ & $19.93 \pm 10.96$ & $12.05 \pm 11.57$ & 0.008 \\
\hline REM sleep (\%TST) & $10.83 \pm 5.73$ & $11.87 \pm 4.26$ & $10.01 \pm 6.62$ & 0.202 \\
\hline Glucose (mg/dL) & $97.40 \pm 30.74$ & $81.77 \pm 9$ & $106.25 \pm 34.9$ & 0.000 \\
\hline Total cholesterol (mg/dL) & $234.11 \pm 46.25$ & $226.13 \pm 46.16$ & $238.62 \pm 46.12$ & 0.240 \\
\hline Triglycerides (mg/dL) & $|92.30 \pm| 38.5 \mid$ & $163.23 \pm 73.03$ & $208.75 \pm 162.84$ & 0.151 \\
\hline HDL-cholesterol (mg/dL) & $41.31 \pm 8.290$ & $42.53 \pm 7.51$ & $40.58 \pm 8.54$ & 0.301 \\
\hline LDL-cholesterol (mg/dL) & $148.85 \pm 42.54$ & $142 \pm 39.29$ & $152.7 \pm 43.63$ & 0.270 \\
\hline
\end{tabular}

Abbreviations: BMI, body mass index; WHR, waist to hip ratio; ESS, Epworth Sleepiness Scale; AHI, Apnea Hypopnea Index; avSpO, average oxyhemoglobin saturation during sleep time; $\mathrm{minSPO}_{2}$, minimum oxyhemoglobin saturation during sleep time; TST, total sleep time; ODI, oxygen desaturation index; REM, rapid eye movement; HDL, high-density lipoprotein; LDL, low density lipoprotein. 
Table 2 Correlations between obstructive sleep apnea and the parameters of metabolic syndrome

\begin{tabular}{|c|c|c|c|}
\hline $\begin{array}{l}\text { Parameters of metabolic } \\
\text { syndrome }\end{array}$ & $\begin{array}{l}\text { Estimated } \\
\text { logistic } \\
\text { coefficient }\end{array}$ & Wald test & $P$ \\
\hline $\begin{array}{l}\text { Waist circumference exceeding } \\
102 \mathrm{~cm} \text { (males) or } \\
88 \mathrm{~cm} \text { (females) }\end{array}$ & 0.708 & 1.250 & 0.264 \\
\hline $\begin{array}{l}\text { Triglycerides level exceeding } \\
150 \mathrm{mg} / \mathrm{dL}\end{array}$ & -0.313 & 0.361 & 0.548 \\
\hline $\begin{array}{l}\text { HDL-cholesterol level below } \\
40 \mathrm{mg} / \mathrm{dL} \text { (males) or } \\
50 \mathrm{mg} / \mathrm{dL} \text { (females) }\end{array}$ & 0.393 & 0.594 & 0.441 \\
\hline $\begin{array}{l}\text { Glucose levels exceeding } \\
110 \mathrm{mg} / \mathrm{dL}\end{array}$ & 2.530 & 9.906 & 0.002 \\
\hline $\begin{array}{l}\text { Blood pressure exceeding } \\
\text { I } 30 \mathrm{mmHg} \text { (systolic) or } \\
85 \mathrm{mmHg} \text { (diastolic) }\end{array}$ & 0.399 & 0.296 & 0.586 \\
\hline
\end{tabular}

between MS and the percentage of slow-wave sleep (SWS) (ie, stages 3 and 4), in total sleep time. Correlations between anthropometrics, sleep characteristics and MS can be seen in Table 3.

Table 3 Correlations between the presence of metabolic syndrome and anthropometric and sleep characteristics of the studied population

\begin{tabular}{|c|c|c|c|}
\hline Characteristic & $\begin{array}{l}\text { Estimated } \\
\text { logistic } \\
\text { coefficient }\end{array}$ & Wald test & $P$ \\
\hline Age (years) & 0.050 & 2.222 & 0.136 \\
\hline BMI $\left(\mathrm{kg} / \mathrm{m}^{2}\right)$ & 0.062 & 1.222 & 0.269 \\
\hline Neck circumference $(\mathrm{cm})$ & 0.248 & 3.461 & 0.063 \\
\hline Waist circumference $(\mathrm{cm})$ & 0.035 & 1.376 & 0.241 \\
\hline Hip circumference $(\mathrm{cm})$ & 0.033 & 1.599 & 0.206 \\
\hline WHR & -2.732 & 0.350 & 0.554 \\
\hline ESS & 0.034 & 0.373 & 0.542 \\
\hline AHI (events/hour) & 0.008 & 0.478 & 0.489 \\
\hline $\mathrm{avSpO}_{2}(\%)$ & -0.037 & 0.270 & 0.604 \\
\hline $\operatorname{minSpO}_{2}(\%)$ & -0.038 & 1.260 & 0.262 \\
\hline $\begin{array}{l}\text { TST with } \mathrm{SpO}_{2} \\
<90 \% \text { (\%TST) }\end{array}$ & 0.011 & 0.850 & 0.356 \\
\hline ODI (events/hour) & 0.011 & 0.853 & 0.356 \\
\hline Sleep efficiency (\%TST) & -0.085 & 2.257 & 0.133 \\
\hline Stage I (\%TST) & 0.017 & 0.120 & 0.729 \\
\hline Stage 2 (\%TST) & 0.047 & 2.294 & 0.130 \\
\hline Stage $3+4$ (\%TST) & -0.079 & 4.699 & 0.030 \\
\hline REM sleep (\%TST) & -0.123 & 3.316 & 0.069 \\
\hline
\end{tabular}

Abbreviations: BMI, body mass index; WHR, waist to hip ratio; ESS, Epworth Sleepiness Scale; AHI, Apnea Hypopnea Index; avSpO${ }_{2}$, average oxyhemoglobin saturation during sleep time; $\operatorname{minSpO}{ }_{2}$, minimum oxyhemoglobin saturation during sleep time; TST, total sleep time; ODI, oxygen desaturation index; REM, rapid eye movement.

\section{Discussion}

This work explores the association between MS and OSA in otherwise-healthy Caucasian male subjects with symptoms indicating sleep-related breathing disorders. Our study shows an association between those two entities. The association between MS and OSA is not a novel finding. MS has already been found significantly higher in OSA patients than the general population..$^{20,23-25,29}$ So far, however, investigation has focused on detecting MS in OSA patients and showing that MS is more frequent in such patients than in the general population. The new aspect that this study is attempting to examine is the reverse association. To the best of our knowledge, this is the first study on otherwise-healthy males who were recruited from an Outpatient Clinic of Obesity, Diabetes and Metabolism and referred because of symptoms suggesting sleep-disordered breathing. In this specific setting, prevalence of MS was, naturally, high (almost 6 out of 10 patients), and its presence was strongly associated with OSA. Of note, this association became marginally nonsignificant after adjustment for BMI. Peled et al reported that BMI did not affect the relationship between the number of MS features and OSA severity. ${ }^{29}$ Nevertheless, their study differed from ours in several ways. First, they did not recruit selected subjects, free from comorbidities, who were regularly attending an Outpatient Obesity Clinic. Secondly, they included both males and females, whereas we confined the enquiry to males. Finally, they examined a metabolic score rather than the presence or not of MS. ${ }^{29}$ Overall, obesity has already been recognized as a potential confounding factor in the association between MS and OSA, that needs further clarification. $^{20,23-25,29}$

Importantly, OSA patients exhibited elevated fasting glucose levels in comparison to age-matched controls. More importantly, increased serum glucose was significantly associated with the presence of OSA. Hyperglycemia was the only component of MS that showed an association with OSA independent of BMI. The increase in serum glucose among patients with OSA concurs with previous findings. . $^{5,25,30}$ Elevated serum glucose is demonstrable in cases of OSA among severely obese subjects, ${ }^{25}$ hypertensive males ${ }^{30}$ and men from the general population. ${ }^{5}$ The independence from BMI has been reported for hypertensive males. ${ }^{30}$ Our findings add to the notion that hyperglycemia is a pivotal diagnostic hallmark in patients with OSA. The novelty of the finding lies in showing a role for hyperglycemia in a different setting, ie, otherwise-healthy males with MS from a specialized metabolic clinic, whose symptoms raised suspicion of sleep-disordered breathing. 
Additionally, MS was associated with altered distribution of sleep stages. Indeed, the percentage of slow-wave sleep out of total sleep time was reduced in subjects with MS. This could indicate inferior sleep quality, although sleep efficiency and the proportion of the other sleep stages remained unaffected. There is growing concern that the suppression of slow-wave sleep may increase the risk for the development of type 2 diabetes. ${ }^{31-33}$ It appears that poor sleep quality perpetuates the activation of the sympathetic nervous system and exacerbates pre-existing dysregulation of the endocrine system. In this context, our finding reinforces the emerging importance of the interaction between sleep disorders and impaired glucose homeostasis, and suggests that this interaction may hold true as early as the stage of MS before the development of type 2 diabetes.

This study used the NCEP ATP III definition of MS ${ }^{17}$ and not the IDF criteria. ${ }^{14}$ While the latter are more recent, most work has been performed with the former. ${ }^{20,21}$ Since the IDF has suggested diagnosing MS at lower thresholds for fasting glucose (ie, $100 \mathrm{mg} / \mathrm{dL}$ ) and waist circumference (94 $\mathrm{cm}$ in men and $80 \mathrm{~cm}$ in women) than the NCEP ATP III $(110 \mathrm{mg} / \mathrm{dL}, 102 \mathrm{~cm}$ in men and $88 \mathrm{~cm}$ in women, respectively), the frequency of MS in OSA patients might be expected to rise. Clearly, the newer IDF criteria offer the opportunity to re-examine the association between MS and OSA. The diversity of criteria reflects the complexity of MS and the ever-growing experience of the subject. ${ }^{34}$ Clearly, re-examination with the newer criteria has the potential to enrich our knowledge in this field.

The limitations of the present work may be outlined as follows. First, it recruited male subjects only, and so its findings cannot be applied to females. However, there appears to be a different effect of sleep apnea on metabolic profile in women, partly, at least, due to different hormonal regulation, ${ }^{35,36}$ and so we chose to focus on male subjects. Secondly, our study population is small, and its findings need to be viewed with caution. For instance, it is surprising, from a clinical point of view, that blood pressure and waist circumference were not associated with the presence of OSA. The same holds true for the association of slow-wave sleep with MS. Although the suppression of slow wave sleep has been identified as a risk factor for future diabetes, ${ }^{31-33}$ the precise mechanism underlying the association with MS would benefit from further clarification. These issues need to be re-examined in a larger patient series. Furthermore, there are no follow-up data to show the impact of the components of MS for the long-term risk of OSA. Obviously, this was beyond the scope of this work, which had a cross-sectional design. Finally, we did not quantify insulin resistance (eg, by the Homeostatic Model Assessment), but we aimed to evaluate the utility of very simple metabolic parameters as used in everyday practice for the detection of OSA.

This study may have the following practical implications. Among men with MS, free from comorbidities, who report symptoms suggestive of sleep-disordered breathing, high serum glucose is associated with a significant increase in the frequency of OSA, even after adjustment for BMI. Moreover, our results point to disordered-sleep architecture, with impaired sleep quality in the presence of MS. Arguably, it appears useful for the clinician treating men with diagnosed MS to bear in mind that they may have some reduction of sleep quality and that hyperglycemia increases the likelihood of overt OSA. Given that OSA itself aggravates insulin resistance $e^{3,5,9,20,21}$ and tends to increase cardiovascular morbidity, ${ }^{2,12}$ early specialist referral for diagnosis and management of this condition might be anticipated to be beneficial. This argument is enhanced by the recent recognition that the risk of OSA needs to be addressed in patients with frank type 2 diabetes, a condition of more profoundly impaired glucose metabolism. ${ }^{37}$

In conclusion, among male subjects with MS who have symptoms indicative of sleep-disordered breathing, elevated serum glucose levels increase the likelihood for the presence of OSA. Moreover, there is a significant negative correlation between MS and the percentage of slow-wave sleep in total sleep time, indicating poorer sleep quality in the presence of MS. These results provide further evidence for the association between OSA and MS. Further work is now needed to shed more light on the pathophysiology of this bidirectional association, as well as to ascertain whether glucose and/or other components of MS can be meaningfully used for appropriate referral and early detection of OSA in such patients.

\section{Disclosures}

The authors report no conflicts of interest in this work.

\section{References}

1. Young T, Palta M, Dempsey J, et al. The occurrence of sleepdisordered breathing among middle-aged adults. $N$ Engl J Med. 1993;328(17):1230-1235.

2. McNicholas WT, Bonsigore MR. Sleep apnoea as an independent risk factor for cardiovascular disease: current evidence, basic mechanisms and research priorities. Eur Respir J. 2007;29(1):156-178.

3. Ip MS, Lam B, Ng MM, Lam WK, Tsang KW, Lam KS. Obstructive sleep apnea is independently associated with insulin resistance. Am J Respir Crit Care Med. 2002;165(5):670-676.

4. Punjabi NM, Sorkin JD, Katzel LI, Goldberg AP, Schwartz AR, Smith PL. Sleep-disordered breathing and insulin resistance in middleaged and overweight men. Am J Respir Crit Care Med. 2002;165(5) $677-682$. 
5. Punjabi NM, Shahar E, Redline S, Gottlieb DJ, Givelber R, Resnick HE. Sleep-disordered breathing, glucose intolerance, and insulin resistance: the Sleep Heart Health Study. Am J Epidemiol. 2004;160(6): 521-530.

6. Ciftci TU, Kokturk O, Bukan N, Bilgihan A. The relationship between serum cytokine levels with obesity and obstructive sleep apnea syndrome. Cytokine. 2004;28(2):87-91.

7. Minoguchi K, Tazaki T, Yokoe T, et al. Elevated production of tumor necrosis factor-alpha by monocytes in patients with obstructive sleep apnea syndrome. Chest. 2004;126(5):1473-1479.

8. Reichmuth KJ, Austin D, Skatrud JB, Young T. Association of sleep apnea and type II diabetes: a population-based study. Am J Respir Crit Care Med. 2005;172(12):1590-1595.

9. Shin C, Kim J, Lee S, et al. Association of habitual snoring with glucose and insulin metabolism in nonobese Korean adult men. Am J Respir Crit Care Med. 2005;171(3):287-291.

10. Ryan S, Taylor CT, McNicholas WT. Predictors of elevated nuclear factor-kappaB-dependent genes in obstructive sleep apnea syndrome. Am J Respir Crit Care Med. 2006;174(7):824-830.

11. Pack AI. Advances in sleep-disordered breathing. Am J Respir Crit Care Med. 2006;173(1):7-15.

12. Ryan S, Taylor CT, McNicholas WT. Systemic inflammation: a key factor in the pathogenesis of cardiovascular complications in obstructive sleep apnoea syndrome? Thorax. 2009;64(7):631-636.

13. Eckel RH, Grundy SM, Zimmet PZ. The metabolic syndrome. Lancet. 2005;365(9468):1415-1428.

14. Alberti KG, Zimmet P, Shaw J. The metabolic syndrome: a new worldwide definition. Lancet. 2005;366(9491):1059-1062.

15. Kostapanos MS, Liamis GL, Elisaf M. Features of the metabolic syndrome relating to cardiorenal outcomes. Arch Med Sci. 2008;4(4):424-426.

16. Ford ES, Giles WH, Dietz WH. Prevalence of the metabolic syndrome among US adults: findings from the third National Health and Nutrition Examination Survey. JAMA. 2002;287(3):356-359.

17. Expert Panel on Detection, Evaluation, and Treatment of High Blood Cholesterol in Adults. Executive summary of the third report of the National Cholesterol Education Program (NCEP) expert panel on detection, evaluation, and treatment of high blood cholesterol in adults (Adult Treatment Panel III). JAMA. 2001;285(19):2486-2497.

18. Tasali E, Ip MSM. Obstructive sleep apnea and metabolic syndrome: Alterations in glucose metabolism and inflammation. Proc Am Thorac Soc. 2008;5(2):207-217.

19. Miller MA, Cappuccio FP. Inflammation, sleep, obesity and cardiovascular disease. Curr Vasc Pharmacol. 2007;5(2):93-102.

20. Coughlin SR, Mawdsley L, Mugarza JA, et al. Obstructive sleep apnoea is independently associated with an increased prevalence of metabolic syndrome. Eur Heart J. 2004;25(9):735-741.

21. Vgontzas AN, Bixler EO, Chrousos GP. Sleep apnea is a manifestation of the metabolic syndrome. Sleep Med Rev. 2005;9(3):211-224.

22. Vgontzas AN, Bixler EO, Chrousos GP. Metabolic disturbances in obesity versus sleep apnoea: the importance of visceral obesity and insulin resistance. J Intern Med. 2003;254(1):32-44.
23. Gruber A, Horwood F, Sithole J, Ali NJ, Idris I. Obstructive sleep apnoea is independently associated with the metabolic syndrome but not insulin resistance state. Cardiovasc Diabetol. 2006;5:22.

24. Lam JC, Lam B, Lam CL, et al. Obstructive Sleep Apnea and the metabolic syndrome in community-based Chinese adults in Hong Kong. Respir Med. 2006;100:980-987.

25. Sasanabe R, Banno K, Otake K, et al. Metabolic syndrome in Japanese patients with obstructive sleep apnea syndrome. Hypertens Res. 2006;29(5):315-322.

26. The Expert Committee on the Diagnosis and Classification of Diabetes Mellitus. Report of the Expert Committee on the Diagnosis and Classification of Diabetes Mellitus. Diabetes Care. 1997;20(7): 1183-1197.

27. Joint National Committee on Prevention, Detection, Evaluation, and Treatment of High Blood Pressure. The seventh report of the Joint National Committee on Prevention, Detection, Evaluation and Treatment of High Blood Pressure: the JNC 7 report. JAMA. 2003;289(19):2560-2572.

28. Tsara V, Serasli E, Amfilochiou A, Constantinidis T, Christaki P. Greek version of the Epworth Sleepiness Scale. Sleep Breath. 2004;8(2): 91-95.

29. Peled N, Kassirer M, Shitrit D, et al. The association of OSA with insulin resistance, inflammation and metabolic syndrome. Respir Med. 2007;101(8):1696-1701.

30. Grunstein RR, Stenlöf K, Hedner J, Sjöström L. Impact of obstructive sleep apnea and sleepiness on metabolic and cardiovascular risk factors in the Swedish Obese Subjects (SOS) Study. Int J Obes Relat Metab Disord. 1995;19(6):410-418.

31. Elmasry A, Lindberg E, Berne C, et al. Sleep-disordered breathing and glucose metabolism in hypertensive men: a population-based study. J Intern Med. 2001;249(2):153-161.

32. Van Cauter E, Holmback U, Knutson K, et al. Impact of sleep and sleep loss on neuroendocrine and metabolic function. Horm Res. 2007;67 Suppl 1:2-9.

33. Tasali E, Leproult R, Spiegel K. Reduced sleep duration or quality: relationships with insulin resistance and type 2 diabetes. Prog Cardiovasc Dis. 2009;51(5):381-391.

34. Spiegel K, Tasali E, Leproult R, Van Cauter E. Effects of poor and short sleep on glucose metabolism and obesity risk. Nat Rev Endocrinol. 2009;5(5):253-261.

35. Lévy P, Bonsignore MR, Eckel J. Sleep, sleep-disordered breathing and metabolic consequences. Eur Respir J. 2009;34(1):243-260.

36. Miller MA, Kandala NB, Kivimaki J, et al. Gender differences in the cross-sectional relationships between sleep duration and markers of inflammation: Whitehall II Study. Sleep. 2009;32(7):857-864.

37. Tasali E, Mokhlesi B, Van Cauter E. Obstructive sleep apnea and type 2 diabetes: interacting epidemics. Chest. 2008;133(2):496-506.
Vascular Health and Risk Management

\section{Publish your work in this journal}

Vascular Health and Risk Management is an international, peerreviewed journal of therapeutics and risk management, focusing on concise rapid reporting of clinical studies on the processes involved in the maintenance of vascular health; the monitoring, prevention and treatment of vascular disease and its sequelae; and the involvement of

\section{Dovepress}

metabolic disorders, particularly diabetes. This journal is indexed on PubMed Central and MedLine. The manuscript management system is completely online and includes a very quick and fair peer-review system, which is all easy to use. Visit http://www.dovepress.com/ testimonials.php to read real quotes from published authors. 